\title{
Slope Optimal Designs for Third Degree Kronecker Model Mixture Experiments
}

\author{
Cheruiyot Kipkoech ${ }^{1}$, Koske Joseph ${ }^{2}$, Mutiso John ${ }^{2}$ \\ ${ }^{1}$ Department of Mathematics and Physical Sciences, Maasai Mara University, Narok, Kenya \\ ${ }^{2}$ Department of Statistics and Computer Science, Moi University, Eldoret, Kenya
}

Email address:

cherukipkoech@gmail.com (C. Kipkoech)

\section{To cite this article:}

Cheruiyot Kipkoech, Koske Joseph, Mutiso John. Slope Optimal Designs for Third Degree Kronecker Model Mixture Experiments. American Journal of Theoretical and Applied Statistics. Vol. 6, No. 4, 2017, pp. 170-175. doi: 10.11648/j.ajtas.20170604.11

Received: October 13, 2016; Accepted: October 28, 2016; Published: June 1, 2017

\begin{abstract}
Mixture experiments are special type of response surface designs where the factors under study are proportions of the ingredients of a mixture. In response surface designs the main interest of the experimenter may not always be in the response at individual locations, but the differences between the responses at various locations is of great interest. Most of the studies on estimation of slope (rate of change) have concentrated in Central Composite Designs (CCD) yet mixture experiments are intended to show the response for all possible formulations of the mixture and to identify optimal proportions for each of the ingredients at different locations. Slope optimal mixture designs for third degree Kronecker model were studied in order to obtained optimal formulations for all possible ingredients in simplex centroid. Weighted Simplex Centroid Designs (WSCD) and Uniformly Weighted Simplex Centroid Designs (UWSCD) mixture experiments were obtained in order to identify optimal proportions for each of the ingredients formulation. Derivatives of the Kronecker model mixture experiment were used to obtain Slope Information Matrices (SIM) for four ingredients. Maximal parameters of interest for third degree Kronecker model were considered. D-, E-, A-, and T- optimal criteria and their efficiencies for both WSCD and UWSCD third degree Kronecker model were obtained. UWSCD was found to be more efficient than WSCD for almost all the points in the simplex designs, therefore recommended for more optimal results in mixture experiments.
\end{abstract}

Keywords: Kronecker Model, Optimal Designs, Slope Information Matrices (SIM), Weighted Simplex Centroid Designs, A-, D-, E- and T-Optimality

\section{Introduction}

Response surface methodology (RSM) is a collection of mathematical and statistical tools or techniques that are useful for modeling and analysis of problems in which a response of interest is influence by several ingredients and the objective is to optimize this response, Montgomery (2001). Response Surface Methodology is an important subject in the statistical design and analysis of experiments. Mixture experiments are special type of RSM associated with the investigation of the $\mathrm{m}$ factors, assumed to influence the response only through proportions in which they are blended together. The mixture ingredients $\mathrm{t}_{1}, \mathrm{t}_{2}, \ldots, \mathrm{t}_{\mathrm{m}}$ are such that $\mathrm{t}_{\mathrm{i}} \geq 0$ and further restricted by $\sum t_{i}=1$. Thus the experimental domain is the probability simplex

$$
T_{m}=\left\{t=\left(t_{1}, \ldots, \quad t_{m},\right)^{\prime} \in[0,1]^{m}: \sum_{i=1}^{m} t_{i}=1\right\}
$$

Under experimental condition $t \in T_{m}$, the response $Y_{t}$ is taken to be a real-valued random variable. In a polynomial regression model the expected value $E\left(Y_{t}\right)$ is a polynomial function of $t$. The work done by Draper and Pukelsheim (1998) is being extended to polynomial regression model for third degree mixture model. Korir et al (2008) extended the work to third degree Kronecker model by use of equivalent theorem in calculation of weights, also Kerich et al (2014) studied the D-optimal designs for third degree Kronecker model mixture experiments with application in artificial sweetener experiment. In many applications of response surface methodology, good estimation of the derivatives of the response function is as important as estimation of the 
mean response. From the work of Hader and Park (1978), Huda and Al-Siha (1999) and Huda (2006), it is clear that most of the work has been done on central composite designs hence there was a need to extend the concept of slope to mixture experiments third degree Kronecker, this method was therefore used for proper identification of the ingredients ratio that leads to an optimal response.

The S-polynomial is given as,

$$
E\left(Y_{t}\right)=f(t)^{\prime} \theta=\sum_{i=1}^{m} \theta_{i} t_{i}+\sum_{\substack{i, j=1 \\ i<j}}^{m} \theta_{i j} t_{i} t_{j}+\sum \sum \sum_{i<j<k}^{m} \theta_{i j k} t_{i} t_{j} t_{k}
$$

and the homogeneous third-degree K-polynomial is

$$
E\left(Y_{t}\right)=f(t)^{\prime} \theta=(t \otimes t \otimes t)^{\prime} \theta=\sum_{i=1}^{m} \sum_{j=1}^{m} \sum_{k=1}^{m} \theta_{i j k} t_{i} t_{j} t_{k}
$$

in which the Kronecker powers $t^{\otimes^{3}}=(t \otimes t \otimes t),\left(\mathrm{m}^{3}+1\right)$ vectors, consists of pure cubic and three-way interactions of components of $t$ in lexicographic order of the subscripts and with evident that third-degree restrictions are $\theta_{i j k}=\theta_{i k l}=\theta_{j i k}=\theta_{j k i}=\theta_{k i j}=\theta_{k j i}$ for all $i, j$ and $\mathrm{k}$

All observations taken in an experiment are assumed to be of equal unknown variance and uncorrelated. The moment matrix $M(\eta)=\sum_{j=1}^{t} w_{j}\left(t_{j}\right) f\left(t_{j}\right)^{t}=\int_{\eta} f(t) f(t)^{t} d \eta$ for the third degree Kronecker model has all entries homogeneous in degree six and reflects the statistical properties of a design $\eta$. The moment matrix can be partition into sub moments according to the number of ingredients in a simplex centroid design as follows

$$
M(\eta)=\alpha_{1} M\left(\eta_{1}\right)+\alpha_{2} M\left(n_{2}\right)+\ldots+\alpha_{m} M\left(n_{m}\right)
$$

For Uniformly Weighted Simplex Centroid Designs (UWSCD), the weights are assumed to be distributed uniformly in the sub moments matrices. Hence $\alpha_{1}=\alpha_{2}=\ldots=\alpha_{m}=\frac{1}{m}$ and their moment matrix is given as,

$$
M(\eta)=\frac{1}{m} M\left(\eta_{1}\right)+\frac{1}{m} M\left(n_{2}\right)+\ldots+\frac{1}{m} M\left(n_{m}\right)
$$

\section{Information Matrix}

Consider the Euclidean unit vectors in $\mathfrak{R}^{m}$ denoted by $e_{1}, e_{2}, \ldots, e_{m}$ and the set for

$$
e_{i i j}=e_{i} \otimes e_{i} \otimes e_{j}, \mathrm{e}_{i j k}=e_{i} \otimes e_{j} \otimes e_{k} \text { for } \mathrm{i}<\mathrm{j}<\mathrm{k}, \mathrm{i}, \mathrm{j}, \mathrm{k}=\{1,2, \ldots, \mathrm{m}\}
$$

Let $\mathrm{K}$ be a $k \times s$ coefficient matrix such that

$$
K=\left(K_{1} ; K_{2} ; K_{3}\right) \in \Re^{m^{3} \times(m+1)}
$$

where

$$
K_{1}=\sum_{i=1}^{m} e_{i i i} e_{i}^{\prime}, \quad K_{2}=\frac{1}{3(m-1)}\left[\sum_{\substack{i, j=1 \\ i<j}}^{m}\left(e_{i i j}+e_{i j i}+e_{j i i}\right) e_{i}^{\prime}\right] \quad \text { and } \quad K_{3}=\frac{1}{m(m-1)(m-2)} \sum_{\substack{i, j, k=1 \\ i \neq j \neq k}}^{m}\left(e_{i j k}\right)
$$

The Kronecker model of the full parameter vector $\theta \in \mathfrak{R}^{m^{3}}$ is not estimable. When fitting this model, the parameter subsystem considered in this study can be written as

$$
K^{\prime} \theta=\left\{\begin{array}{l}
\left(\theta_{i i i}\right)_{1 \leq i \leq m} \\
\frac{1}{3(m-1)}\left\{\sum_{i, j=1}^{m}\left(\theta_{i j j}+\theta_{i j i}+\theta_{j i i}\right)\right.
\end{array}\right\}, \mathfrak{R}^{(m+1)} \text { for all } \theta \in \mathfrak{R}^{m^{3}}
$$

where $K \in \mathfrak{R}^{m^{3} \times(m+1)}$

The parameter subsystem $K^{\prime} \theta$ of interest is a maximal parameter system in the full parameter model. The information matrix for the parameter subsystem is given by

$$
C_{k}(M(\eta))=L M(\eta) L \in N N D(s)
$$

where $\mathrm{L}$ is the left inverse of coefficient matrix $\mathrm{K}$ and is defined by

$$
L=\left(K K^{-1} K^{`}\right.
$$

Thus the information matrices for $K^{\wedge} \theta$ are linear transformation of moment matrices.

\section{Application in Four Factors Mixture Experiments}

Using simplex restrictions, we obtained the optimal weights for WSCD, $\alpha_{1}, \alpha_{2}, \alpha_{3}$ and $\alpha_{4}$ as $4 / 15,6 / 15,4 / 15$ and $1 / 15$ respectively. From equation (4), we have the moment matrix for four factors mixture experiments as,

$$
M(\eta)=\frac{4}{15} M\left(\eta_{1}\right)+\frac{6}{15} M\left(n_{2}\right)+\frac{4}{15} M\left(n_{3}\right)+\frac{1}{15} M\left(n_{4}\right)
$$

where,

$$
M\left(\eta_{1}\right)=\frac{1}{4}\left[e_{111}\left(e_{111}\right)^{\prime}+e_{222}\left(e_{222}\right)^{\prime}+e_{333}\left(e_{333}\right)^{\prime}+e_{444}\left(e_{444}\right)^{\prime}\right]
$$

$$
\begin{aligned}
M\left(\eta_{2}\right)=\frac{1}{384} & {\left[\left(d_{1} \otimes d_{1} \otimes d_{1}\right)\left(d_{1} \otimes d_{1} \otimes d_{1}\right)^{\prime}+\left(d_{2} \otimes d_{2} \otimes d_{2}\right)\left(d_{2} \otimes d_{2} \otimes d_{2}\right)^{\prime}\right.} \\
& +\left(d_{3} \otimes d_{3} \otimes d_{3}\right)\left(d_{3} \otimes d_{3} \otimes d_{3}\right)^{\prime}+\left(d_{4} \otimes d_{4} \otimes d_{4}\right)\left(d_{4} \otimes d_{4} \otimes d_{4}\right)^{\prime} \\
& \left.+\left(d_{5} \otimes d_{5} \otimes d_{5}\right)\left(d_{5} \otimes d_{5} \otimes d_{5}\right)^{\prime}+\left(d_{6} \otimes d_{6} \otimes d_{6}\right)\left(d_{6} \otimes d_{6} \otimes d_{6}\right)^{\prime}\right]
\end{aligned}
$$




$$
\begin{gathered}
M\left(\eta_{3}\right)=\frac{1}{2916}\left[\left(f_{1} \otimes f_{1} \otimes f_{1}\right)\left(f_{1} \otimes f_{1} \otimes f_{1}\right)^{\prime}+\left(f_{2} \otimes f_{2} \otimes f_{2}\right)\left(f_{2} \otimes f_{2} \otimes f_{2}\right)^{\prime}\right. \\
\left.+\left(f_{3} \otimes f_{3} \otimes f_{3}\right)\left(f_{3} \otimes f_{3} \otimes f_{3}\right)^{\prime}+\left(f_{4} \otimes f_{4} \otimes f_{4}\right)\left(f_{4} \otimes f_{4} \otimes f_{4}\right)^{\prime}\right] \\
M\left(\eta_{4}\right)=\frac{1}{4096} J_{64}
\end{gathered}
$$

also,

$$
\begin{gathered}
d_{1}=\left(1_{4}-e_{4}-e_{3}\right), d_{2}=\left(1_{4}-e_{2}-e_{4}\right), d_{3}=\left(1_{4}-e_{1}-e_{4}\right), d_{4}=\left(1_{4}-e_{1}-e_{3}\right), \\
d_{4}=\left(1_{4}-e_{1}-e_{3}\right), d_{5}=\left(1_{4}-e_{1}-e_{2}\right), d_{6}=\left(1_{4}-e_{2}-e_{3}\right) \\
f_{1}=\left(1_{4}-e_{4}\right), f_{2}=\left(1_{4}-e_{2}\right), f_{3}=\left(1_{4}-e_{3}\right), f_{4}=\left(1_{4}-e_{1}\right),
\end{gathered}
$$$$
e_{1}=\left(\begin{array}{l}
1 \\
0 \\
0 \\
0
\end{array}\right), e_{2}=\left(\begin{array}{l}
0 \\
1 \\
0 \\
0
\end{array}\right), e_{3}=\left(\begin{array}{l}
0 \\
0 \\
1 \\
0
\end{array}\right) \text { and } e_{4}=\left(\begin{array}{l}
0 \\
0 \\
0 \\
1
\end{array}\right)
$$

The Uniformly Weighted Simplex Centriod Designs (UWSCD) for four ingredients were assumed to assign uniform weights to the four elementary centroid designs, $n_{1}, n_{2}, n_{3}$ and $n_{4}$, such that all weights are equal. That is,

$$
\alpha_{1}=\alpha_{2}=\alpha_{3}=\alpha_{4}=0.25
$$

The moment matrix for uniformly weighted simplex centriod designs in (5) for four ingredients is given by

$$
\begin{aligned}
& M(\eta)=0.25 M\left(\eta_{1}\right)+0.25 M\left(n_{2}\right)+0.25 M\left(n_{3}\right)+0.25 M\left(n_{3}\right)(17) \\
& K_{1}=\sum_{i=1}^{4} e_{i i i} e_{i}{ }^{\prime}=e_{111} e_{1}{ }^{\prime}+e_{222} e_{2}{ }^{\prime}+e_{333} e_{3}{ }^{\prime}++e_{444} e_{4}{ }^{\prime} \\
& K_{2}=\frac{1}{9}\left\{\sum_{\substack{i j=1 \\
i \neq j}}^{3}\left(e_{i j}+e_{i j i}+e_{j i i}\right) e_{i}{ }^{\prime}\right\} \\
& =\frac{1}{9}\left\{\left(e_{112}+e_{121}+e_{211}\right) e_{1}{ }^{\prime}+\left(e_{122}+e_{212}+e_{221}\right) e_{2}{ }^{\prime}+\left(e_{133}+e_{313}+e_{331}\right) e_{3}{ }^{\prime}+\left(e_{144}+e_{414}+e_{441}\right) e_{4}{ }^{\prime}\right\}
\end{aligned}
$$

where,

$M\left(\eta_{1}\right), M\left(n_{2}\right), M\left(n_{3}\right)$ and $M\left(n_{4}\right)$ are given in (12), (13), (14) and (15) respectively.

The coefficient matrix $\mathrm{K}$ for the four ingredients parameter subsystems of interest in (7) and (8) is given as

$$
K=\left(\mathrm{K}_{1}, K_{2}, K_{3}\right)
$$

where

and

$$
\begin{aligned}
& K_{3}=\frac{1}{24}\left\{\sum_{\substack{i k=1 \\
i \neq j \neq k}}^{4} e_{i j k}\right\} \\
& =\frac{1}{24}\left(e_{123}+e_{132}+e_{231}+e_{213}+e_{312}+e_{321}+e_{124}+e_{134}+e_{142}+e_{143}+e_{214}+e_{234}\right. \\
& \left.\quad+e_{241}+e_{243}+e_{314}+e_{324}+e_{341}+e_{342}+e_{412}+e_{413}+e_{421}+e_{423}+e_{431}+e_{432}\right)
\end{aligned}
$$

The left inverse $\mathrm{L}$ in (10) for four ingredients is given as,

$$
L^{\prime}=\left(\mathrm{K}_{1}, 6 K_{2}, 24 K_{3}\right)
$$

\section{Slope Designs}

In response surface designs the main interest of the experimenter may not always be in the response at individual locations but, the differences between the responses at various locations may be of greater interest, Herzberg (1967),
Box and Draper (1980), Huda and Mukerjee (1984) and Huda (2006).

We know that to maximize the response, the movement of the design center must be in the direction of the directional derivatives of the response function, that is, $\frac{\partial Y_{t}}{\partial t}$. Let $\mathrm{H}$ be a matrix arising from the differentiation of $f(t)^{\prime} \theta$ with respect to each of the $m$ independent factors, Sung. et al (2009). That is; 


$$
\begin{aligned}
& H=\left(\frac{\partial f^{\prime}(t)}{\partial t_{1}}, \frac{\partial f^{\prime}(t)}{\partial t_{2}}, \ldots, \frac{\partial f^{\prime}(t)}{\partial t_{m}}\right)^{\prime}
\end{aligned}
$$

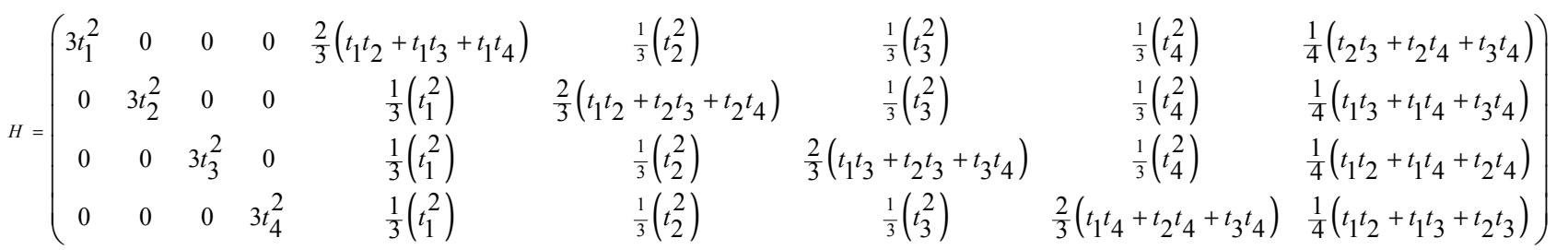

$$
\begin{aligned}
& \text { Therefore, }
\end{aligned}
$$

Using (11) and (19), we obtained the information matrix for Weighted Simplex Centroid Designs (WSCD) as

$$
C_{2}=\left(\begin{array}{ccc}
c_{11} & c_{12} & c_{13} \\
c_{12} & c_{22} & c_{23} \\
c_{13}^{\prime} & c_{23}^{\prime} & c_{33}
\end{array}\right)
$$

where,

$$
\begin{gathered}
c_{11}=x_{11} I_{4}+y_{11} J_{4} ; \quad x_{11}=688.4 \times 10^{-4}, \quad y_{11}=12.40842 \times 10^{-4} \\
c_{12}=x_{12} I_{4}+y_{11} J_{4} ; x_{12}=67.98697 \times 10^{-4}, \quad y_{12}=43.68878 \times 10^{-4} \\
c_{22}=x_{22} I_{4}+y_{22} J_{4} ; x_{22}=220.42181 \times 10^{-4}, \quad y_{22}=172.77722 \times 10^{-4} \\
c_{13}=x_{13} I_{4} ; x_{13}=20.36716 \times 10^{-4} \\
c_{23}=x_{23} I_{4} ; x_{23}=133.92168 \times 10^{-4} \\
c_{33}=x_{22} ; x_{22}=225.43724 \times 10^{-4}
\end{gathered}
$$

The derivative matrix (21) together with information matrix (22) were used to obtain Slope Information Matrices (SIM) at different points of the simplex. At $(1,0,0,0)$, the slope information matrix is given as,

$$
\operatorname{SIM}_{41}=\left(\begin{array}{cc}
6307.40393 \times 10^{-4} & 111.67574 \times 10^{-4}\left(1_{3}{ }^{\prime}\right) \\
111.67574 \times 10^{-4}\left(1_{3}\right) & 43.6887 \times 10^{-4} J_{3}
\end{array}\right)
$$

At the binary blend point $(0.5,0.5,0,0)$ the slope information matrix is of the form

$$
\operatorname{SIM}_{42}=\left(\begin{array}{cc}
399.23626 \times 10^{-4}\left(I_{2}\right)+48.7828 \times 10^{-4} J_{2} & 25.5644 \times 10^{-4} J_{2} \\
25.5644 \times 10^{-4} J_{2} & 13.14636 \times 10^{-4} J_{2}
\end{array}\right)
$$

At the point $(0.333,0.333,0.333,0)$ the slope information matrix is

$$
\operatorname{SIM}_{43}=\left(\begin{array}{cc}
84.2478 \times 10^{-4}\left(I_{3}\right)+22.98775 \times 10^{-4} J_{3} & 12.51869 \times 10^{-4}\left(1_{3}\right) \\
12.51869 \times 10^{-4}\left(1_{3}^{\prime}\right) & 7.0857 \times 10^{-4}
\end{array}\right)
$$

At the point $(1 / 4,1 / 4,1 / 4,1 / 4)$, the slope information matrix is given as

$$
S I M_{44}=\left(\begin{array}{llll}
549 / 125029 & 61156 / 30546869 & 4185 / 2854654 & 4185 / 2854654 \\
61156 / 30546869 & 3764 / 639087 & 5241 / 2207668 & 61156 / 30546869 \\
4185 / 2854654 & 5241 / 2207668 & 549 / 125029 & 4185 / 2854654 \\
4185 / 2854654 & 61156 / 30546869 & 4185 / 2854654 & 549 / 125029
\end{array}\right)
$$

Using (17) and (19), we obtained the information matrix for uniform weighted simplex centroid designs as,

$$
C_{2 u}=\left(\begin{array}{ccc}
c_{11} & c_{12} & c_{13} \\
c_{21} & c_{22} & c_{23} \\
c_{13}^{\prime} & c_{23}^{\prime} & c_{33}
\end{array}\right)
$$


where $c_{11}, c_{12}, c_{13}, c_{22}, c_{23}$ and $c_{33}$ are given as

$$
\begin{gathered}
c_{11}=x_{11} I_{4}+y_{11} J_{4} ; x_{11}=638.8782 \times 10^{-4}, y_{11}=8.8354 \times 10^{-4} \\
c_{12}=c_{21}^{\prime}=x_{12} I_{4}+y_{11} J_{4} ; x_{12}=44.2062 \times 10^{-4}, y_{12}=35.3124 \times 10^{-4} \\
c_{22}=x_{22} I_{4}+y_{22} J_{4} ; x_{22}=148.05 \times 10^{-4}, y_{22}=169.7616 \times 10^{-4} \\
c_{13}=c_{31}^{\prime}=x_{13} 1_{4} ; x_{13}=30.0810 \times 10^{-4} \\
c_{23}=c_{32}^{\prime}=x_{23} 1_{4} ; x_{23}=224.4348 \times 10^{-4} \\
c_{33}=x_{22} ; x_{22}=475.0416 \times 10^{-4}
\end{gathered}
$$

The derivative matrix (21) together with information matrix (27) were used to obtain Slope Information Matrices (SIM) at different points of the UWSCD. At point $(1,0,0,0)$, the slope information matrix is given by,

$$
\operatorname{SIM}_{41 u}=\left(\begin{array}{cc}
5829.4224 \times 10^{-4} & 79.5186 \times 10^{-4}\left(1_{3}^{\prime}\right) \\
79.5186 \times 10^{-4}\left(1_{3}\right) & 35.3124 \times 10^{-4} J_{3}
\end{array}\right)
$$

For binary blends at points $(1 / 2,1 / 2,0,0)$, we have information matrix as,

$$
\operatorname{SIM}_{42 u}=\left(\begin{array}{cc}
365.92289 \times 10^{-4} I_{2}+38.46044 \times 10^{-4} J_{2} & 22.25155 \times 10^{-4} J_{2} \\
22.25155 \times 10^{-4} J^{\prime}{ }_{2} & 13.30321 \times 10^{-4} J_{2}
\end{array}\right)
$$

We obtained slope information matrix at the ternary point $(0.333,0.333,0.333,0)$ as,

$$
\operatorname{SIM}_{43 u}=\left(\begin{array}{cc}
76.08877 \times 10^{-4}\left(I_{3}\right)+21.21016 \times 10^{-4} J_{3} & 14.0479 \times 10^{-4}\left(1_{3}\right) \\
14.0479 \times 10^{-4}\left(1_{3}^{\prime}\right) & 10.16018 \times 10^{-4}
\end{array}\right)
$$

At the central point $(1 / 4,1 / 4,1 / 4,1 / 4)$ of the simplex centroid design, we have the slope information matrix given as,

$$
S I M_{44 u}=\left(\begin{array}{llll}
0.004131819 & 0.002094689 & 0.001552438 & 0.001552438 \\
0.002094689 & 0.005561169 & 0.002341674 & 0.002094689 \\
0.001552438 & 0.002341674 & 0.004131819 & 0.001552438 \\
0.001552438 & 0.002094689 & 0.001552438 & 0.004131819
\end{array}\right)
$$

\section{Optimal Values for Slope Information Matrices (SIM)}

Optimal designs are experimental designs that are generated based on a particular optimality criterion and are generally optimal only for a specific statistical model. The optimality properties of designs are determined by their moment matrices, Pukelsheim (1993). The amount of

\begin{tabular}{|c|c|c|c|c|c|c|c|c|}
\hline \multirow[b]{2}{*}{ BLENDS } & \multicolumn{4}{|c|}{ WEIGHTED SIMPLEX CENTROID (WSC) } & \multicolumn{4}{|c|}{ UNIFORM WEIGHTED SIMPLEX CENTROID (UWSC) } \\
\hline & D- & E- & & T- & D- & E- & A- & $\mathbf{T}-$ \\
\hline $1,0,0,0$ & 0.0000 & 0.0000 & 0.0000 & 0.1609 & 0.0000 & 0.0000 & 0.0000 & 0.1484 \\
\hline $1 / 2,1 / 2,0,0$ & 0.0000 & 0.0000 & 0.0000 & 0.0229 & 0.0000 & 0.0000 & 0.0000 & 0.0208 \\
\hline $1 / 3,1 / 3,1 / 3,0$ & 0.0046 & 0.0004 & 0.0014 & 0.0082 & 0.0047 & 0.0005 & 0.0019 & 0.0075 \\
\hline $1 / 4,1 / 4,1 / 4,1 / 4$ & 0.0039 & 0.0027 & 0.0035 & 0.0048 & 0.0036 & 0.0024 & 0.0031 & 0.0045 \\
\hline
\end{tabular}
information inherent to $\mathrm{C}_{\mathrm{k}}(\mathrm{M}(\eta))$ is provided by $\varphi_{p}$-criteria with $\mathrm{C}_{\mathrm{k}}(\mathrm{M}(\eta)) \in \mathrm{PD}(\mathrm{m})$, defined by:

$$
\varphi_{p}(C)=\left\{\begin{array}{ccc}
\lambda_{\min }(C) & \text { if } & p=-\infty \\
\operatorname{det}(C)^{\frac{1}{s}} & \text { if } & p=0 \\
{\left[\frac{1}{s} \text { trace } C^{p}\right]^{p}} & \text { if } & p \neq 0, \pm \infty
\end{array}\right.
$$

We obtained the optimal values for both Weighted Simplx Centroid (WSC) designs and Uniform Weighted Simplex Centroid Designs (UWSCD) for four ingredients mixture experiments.

Table 1. Optimal Values for Four Ingredients.

Uniformly Weighted Simplex Centroids Design (UWSCD) was observed to yield more optimal values than Weighted
Simplex Centroid Designs (WSCD) at all points of the simplex centroids mixture experiments. 


\section{Efficiencies for Four Ingredients}

The efficiency of WSCD over UWSCD at different point of the simplex centroid designs were summarized as

Table 2. Efficiencies for Four Ingredients.

\begin{tabular}{lllll}
\hline \multicolumn{5}{c}{ Efficiencies (\%) } \\
\hline BLENDS & D- & E- & A- & T- \\
\hline $1,0,0,0$ & 100 & 100 & 100 & 108.42 \\
$1 / 2,1 / 2,0,0$ & 100 & 100 & 100 & 110.09 \\
$1 / 3,1 / 3,1 / 3,0$ & 97.87 & 80 & 73.68 & 109.33 \\
$1 / 4,1 / 4,1 / 4,1 / 4$ & 108.33 & 112.5 & 112.90 & 106.66 \\
\hline
\end{tabular}

From Table 2 , at $(1,0,0,0)$ and $(1 / 2,1 / 2,0,0)$, there was no difference between the two designs in their D-, E- and Aefficiency. However, UWSCD was $8.42 \%$ and $10.09 \%$ more $\mathrm{T}$ - efficient than WSCD at respective blends. For ternary mixture $(0.333,0.333,0.333,0)$, WSCD was $2.13 \%, 20 \%$ and $26.32 \%$ more D-, E- and A- efficient than UWSCD respectively. It was also observed that WSCD was $9.33 \%$ less T-efficient than UWSCD. At point $(1 / 4,1 / 4,1 / 4,1 / 4)$, UWSCD was $8.33 \%, 12.5 \%, 12.9 \%$ and $6.66 \%$ more D-, E-, A- and Tefficient respectively than WSCD. Generally, the D-, E-, Aand T-optimal values for Uniformly Weighted Simplex Centroid (UWSC) designs were better than those of Weighted Simplex Centroid (WSC) designs for four ingredients.

\section{Conclusion}

It was noted that Uniformly Weighted Simplex Centroid (UWSC) designs were more efficient than Weighted Simplex Centroid (WSC) designs. For more optimal results, the experimenter is advised to allocate weights in the mixture components uniformly. In UWSC design, the centroid point $(1 / 4,1 / 4,1 / 4,1 / 4)$ produced the most efficient results than any other point while WSC design yield the optimal results at point $(0.333,0.333,0.333,0)$ only.

\section{References}

[1] Box, G. E. P. and Draper, N. R. (1980). The variance functions of the difference between two estimated responses, J. Roy. Statist. Soc., B 42, 79-82.

[2] Draper, N. R. and Pukelsheim, F. (1998). Mixture models based on homogeneous polynomials. J. Statist. Plann. Inference 71 303-311.

[3] Hader, R. J. and Park, S. H. (1978). Slope-rotatable central composite designs, Technometrics, 20, 413-417.

[4] Herzberg, A. M. (1967). The behavior of the variance functions of the difference between two responses. J. Roy. Statist. Soc. B, 29, 174-179.

[5] Huda, S. (2006). Minimax designs for the difference between estimated responses for the quadratic model over hypercubic regions. Commun. Statist.- Theory meth.

[6] Huda, S. and Al-Shiha, A. A. (1999). On D-Optimal designs for estimating slope. The Indian Journal of Statistics. 61: B, 3, 488-495.

[7] Huda, S. and Mukerjee, R. (1984). Minimizing the maximum variance of the difference between two estimated responses, Biometrika, 71, 381-385.

[8] Kerich, G., Koske, J., Rutto, M., Korir, B., Ronoh, B., Kinyanjui, J. and Kungu, P. (2014). D-Optimal Designs for Third Degree Kronecker Model Mixture Experiments with An Application to Artificial Sweetener Experiment. IOSR Journal of Mathematics, 10 (6), 32-41.

[9] Korir, B. C. (2008)," Kiefer ordering of simplex designs for third-degree mixture models." P. hd. Thesis Moi University.

[10] Montgomery, M. D. (2001). Design and Analysis of Experiments, $5^{\text {th }}$ ed., John Wiley and Sons, New York.

[11] Pukelsheim, F. (1993). Optimal design of experiments. Wiley, New York.

[12] Sung H. P., Hyang S. J. and Rabindra N. D. (2009). Slope rotatbility of second order response surface regression models with correlated error. Quality technology \& quantitative management 6 , (4) 471-492. 